\title{
A Novel Non-Equidistant Grey GRM(1,1) Model Based on Reciprocal Accumulated Generating
}

\author{
Ruibiao Zou \\ College of Sciences, Hunan Agriculture University, Changsha, China \\ Email: rbzou@163.com
}

Received June 6, 2012; revised July 5, 2012; accepted July 13, 2012

\begin{abstract}
Applying the reciprocal accumulated generating and the reconstruction method of $\operatorname{GRM}(1,1)$ model's background value of non-equidistant sequence based on the exponential trait of grey model and the definition of integral for the problem of lower precision as well as lower adaptability in non-equidistant $\mathrm{GM}(1,1)$ model, the calculation formulas were deduced and a novel non-equidistant GRM $(1,1)$ model generated by reciprocal accumulated generating was put forward. The grey GRM(1,1) model can be used in non-equidistant interval \& equidistant interval time series and has the characteristic of high precision as well as high adaptability. Example validates the practicability and reliability of the proposed model.
\end{abstract}

Keywords: GRM Model; Non-Equidistant Interval; Accumulated Generating; Grey System

\section{Introduction}

Grey model as an important part in grey system theory has been widely used in many fields since Professor J.L. Deng proposed the grey system [1]. Among the models, $\operatorname{GM}(1,1)$ has been greatly concerned and been widely used because of the research characteristics such as the small sample and the poor information, as well as the advantages which is simple and practical [1-6]. Most of the grey system models are based on equidistant sequence, but the original data obtained from the actual work are mostly non-equidistant sequence. So that establishing non-equidistant sequence model has a certain practical and theoretical significance. Sequence spacing was regarded as a multiplier to establish the non-equidistance GM $(1,1)$ model which supposed that there is the linear relationship between data difference and time difference [2], but the result from this model can't be ensured to be consistent with the reality. Function transformation method was adopted to reduce the standard deviation coefficient to take the original sequence as new data sequence and estimate the model parameters, and then $\mathrm{GM}(1,1)$ was set up [3], but there is the complicated calculation. In order to improve the accuracy of the fitting and the predict, a variety of methods constructing the background value were proposed and some nonequidistant $\operatorname{GM}(1,1)$ models were established [4-6]. The model improving the background value based on nonhomogeneous GM(1,1) model was established, in which homogeneous exponent function is used for fitting one- time accumulated generating sequence to obtain the higher accuracy $[4,5]$. But according to the solution form of the whitening differential equations in $\operatorname{GM}(1,1)$, the exponent form of one-time accumulated generating sequence is non-homogeneous, and only after accumulating and reducing it is homogeneous. There produce some error by using the homogeneous exponent function to fit. The optimal calculation formula for background value was deduced using non-homogeneous exponent function to fit one-time accumulated generating sequence and equidistant GM(1,1) model was established [6]. But these models are based on common accumulated or inverse accumulated generation in the process of modeling. For non-negative discrete sequence $\mathbf{X}^{(0)}$, the one-time accumulated generation sequence $\mathbf{X}^{(1)}$ is monotonically increasing. When a curve fits $\mathbf{X}^{(1)}$, it is reasonable that the curve is monotonically increasing. It is $\operatorname{GM}(1,1)$ to predict. If $\mathbf{X}^{(0)}$ itself is monotonically decreasing, $\mathbf{X}^{(1)}$ is monotonically increasing and then the model value $\hat{\mathbf{X}}^{(1)}$ is also increased. When $\hat{\mathbf{X}}^{(1)}$ is regressively generated to the predicted value of the original sequence $\hat{\mathbf{X}}^{(0)}$, there will produce an unreasonable calculation errors. For the original sequence with monotonically decreasing trend, accumulated generation in opposite direction was put forward and GOM $(1,1)$ based on accumulated accumulation in opposite direction was established [7]. $\operatorname{GRM}(1,1)$ based on reciprocal generation was built after proposing reciprocal generation [8]. GRM(1,1) was improved to establish the improved grey model CGRM $(1,1)$ based on reciprocal generation [9]. The models that 
based on reciprocal generation and opposite-direction accumulated generation make the generation sequence $\mathbf{X}^{(1)}$ monotone decreasing, and then fitted $\mathbf{X}^{(1)}$ by using the decreasing monotonically curve to obtain the model value $\hat{\mathbf{X}}^{(1)}$ of $\mathbf{X}^{(1)}$.In this case, the reduction process from $\hat{\mathbf{X}}^{(0)}$ to $\mathbf{X}^{(0)}$ will not produce the unreasonable error and it improves modeling accuracy. But the models in $[8,9]$ are equidistant $\operatorname{GRM}(1,1)$. Based on reciprocal accumulated generation combining with the method establishing background value in [4], this paper deduced the model calculation formulas, and established a non-equidistant grey GRM(1,1) model based on reciprocal accumulated generation. This model with high precision has better practical and theoretical significance. Example validates the practicability and reliability of the proposed model.

\section{Non-Equidistant Grey GRM(1,1) Model}

Definition 1. Supposed the sequence

$$
\mathbf{X}^{(00)}=\left[x^{(00)}\left(t_{1}\right), x^{(00)}\left(t_{2}\right), \cdots, x^{(00)}\left(t_{m}\right)\right],
$$

If $\Delta t_{i}=t_{i}-t_{i-1} \neq$ const, where $i=2, \cdots, m$, then $X^{(00)}$ is called as non-equidistant sequence. Supposed

$$
x^{(0)}\left(t_{k}\right)=\frac{1}{x^{(00)}\left(t_{k}\right)}(k=1,2, \cdots, m),
$$

then

$$
\mathbf{X}^{(0)}=\left[x^{(0)}\left(t_{1}\right), x^{(0)}\left(t_{2}\right), \cdots, x^{(0)}\left(t_{m}\right)\right]
$$

is named for reciprocal sequence of $\mathbf{X}^{(00)}$.

Definition 2. Supposed the sequence

$$
\mathbf{X}^{(1)}=\left[x^{(1)}\left(t_{1}\right), x^{(1)}\left(t_{2}\right), \cdots, x^{(1)}\left(t_{n}\right)\right],
$$

where $x^{(0)}\left(t_{k}\right)=\frac{1}{x^{(00)}\left(t_{k}\right)}, k=1,2, \cdots, m$, if

$$
x^{(1)}\left(t_{1}\right)=x^{(0)}\left(t_{1}\right)
$$

and

$$
x^{(1)}\left(t_{k+1}\right)=x^{(1)}\left(t_{k}\right)+x^{(0)}\left(t_{k+1}\right) \cdot \Delta t_{k+1},
$$

where $k=1, \cdots, m-1$, then $\mathbf{X}^{(1)}$ is called as one-time reciprocal accumulated generation of non-equidistant sequence $\mathbf{X}^{(00)}$, and it is denoted by 1-RAG0.

Supposed the original data sequence

$$
\mathbf{X}^{(00)}=\left[x^{(00)}\left(t_{1}\right), \cdots, x^{(00)}\left(t_{m}\right)\right],
$$

where

$$
x^{(00)}\left(t_{j}\right)(j=1,2, \cdots m)
$$

is the observation value at $t_{j}, \mathrm{~m}$ is the data number, and the sequence $\left[x^{(0)}\left(t_{1}\right), x^{(0)}\left(t_{2}\right), \cdots, x^{(0)}\left(t_{m}\right)\right]$ is non- equidistant, that is, the spacing $t_{j}-t_{j-1}$ is not constant.

In order to establish the model, firstly the original data is reciprocal accumulated one time to generate a new sequence as:

$$
\mathbf{X}^{(1)}=\left[x^{(1)}\left(t_{1}\right), x^{(1)}\left(t_{2}\right), \cdots, x^{(1)}\left(t_{m}\right)\right]
$$

where, $x^{(1)}\left(t_{j}\right)(j=1,2, \cdots, m)$ meets the conditions in the definition 2 , that is,

$$
\begin{aligned}
& x_{i}^{(1)}\left(t_{j}\right) \\
& =\left\{\begin{array}{cc}
x_{i}^{(0)}\left(t_{1}\right)+\sum_{k=2}^{j} x_{i}^{(0)}\left(t_{k}\right)\left(t_{k}-t_{k-1}\right), & (j=2, \cdots, m), \\
x_{i}^{(0)}\left(t_{1}\right), & (j=1),
\end{array}\right.
\end{aligned}
$$

Accounting to one-time reciprocal accumulated generation, a non-equidistant $\operatorname{GRM}(1,1)$ model is established as a first-order grey differential equation

$$
\frac{\mathrm{d} x^{(1)}}{\mathrm{d} t}+a \zeta^{(1)}=b,
$$

where $\zeta^{(1)}$ is the background value. Its whitening differential equation is as

$$
\frac{\mathrm{d} x^{(1)}}{\mathrm{d} t}+a x^{(1)}=b .
$$

The whitening differential equation is integrated in the interval $\left[t_{k}, t_{k+1}\right]$ as

$$
\int_{t_{k}}^{t_{k+1}} \mathrm{~d} x^{(1)}+a \int_{t_{k}}^{t_{k+1}} x^{(1)} \mathrm{d} t=b \int_{t_{k}}^{t_{k+1}} \mathrm{~d} t .
$$

Supposed

$$
z^{(1)}\left(t_{k+1}\right)=\int_{t_{k}}^{t_{k+1}} x^{(1)} \mathrm{d} t
$$

is the background value of $x^{(1)}$ in $\left[t_{k}, t_{k+1}\right]$, Equation (3) is converted as

$$
x^{(0)}\left(t_{k+1}\right) \Delta t_{k+1}+a z^{(1)}\left(t_{k+1}\right)=b \Delta t_{k+1} .
$$

As the result forms of the whitening differential equation is exponential, $x^{(1)}$ can be fitted by the equation as $x^{(1)}(t)=A e^{B \cdot t}$. Supposed that $x^{(1)}$ runs through $\left[t_{k}, x^{(1)}\left(t_{k}\right)\right]$ and $\left[t_{k+1}, x^{(1)}\left(t_{k+1}\right)\right]$, it can be obtained as:

$$
b=\ln \left(\frac{\ln x^{(1)}\left(t_{k+1}\right)-\ln x^{(1)}\left(t_{k}\right)}{\Delta t_{k+1}}\right),
$$

$$
C=\frac{\left[x^{(1)}\left(t_{k}\right)\right]^{\frac{t_{k+1}}{\Delta t_{k+1}}}}{\left[x^{(1)}\left(t_{k}\right)\right]^{\frac{t_{k}}{\Delta t_{k+1}}}} .
$$

So the background value can be structured as 


$$
z^{(1)}\left(t_{k+1}\right)=\frac{x^{(0)}\left(t_{k+1}\right)\left(\Delta t_{k+1}\right)^{2}}{\ln x^{(1)}\left(t_{k+1}\right)-\ln x^{(1)}\left(t_{k}\right)} .
$$

The matrix of Equation (4) is expressed as

$$
\left[\begin{array}{c}
x^{(0)}\left(t_{2}\right) \Delta t_{2} \\
x^{(0)}\left(t_{3}\right) \Delta t_{3} \\
\cdots \\
x^{(0)}\left(t_{n}\right) \Delta t_{m}
\end{array}\right]=\left[\begin{array}{cc}
-z^{(1)}\left(t_{2}\right) & \Delta t_{2} \\
-z^{(1)}\left(t_{3}\right) & \Delta t_{3} \\
\cdots & \cdots \\
-z^{(1)}\left(t_{n}\right) & \Delta t_{n}
\end{array}\right]\left[\begin{array}{l}
a \\
b
\end{array}\right] .
$$

Assumed

$$
\mathbf{Y}=\left[\begin{array}{c}
x^{(0)}\left(t_{2}\right) \Delta t_{2} \\
x^{(0)}\left(t_{3}\right) \Delta t_{3} \\
\cdots \\
x^{(0)}\left(t_{m}\right) \Delta t_{m}
\end{array}\right], \mathbf{B}=\left[\begin{array}{cc}
-z^{(1)}\left(t_{2}\right) & \Delta t_{2} \\
-z^{(1)}\left(t_{3}\right) & \Delta t_{3} \\
\cdots & \ldots \\
-z^{(1)}\left(t_{m}\right) & \Delta t_{n}
\end{array}\right], \boldsymbol{\Phi}=\left[\begin{array}{l}
a \\
b
\end{array}\right],
$$

where, $\boldsymbol{\Phi}$ is parameter vector to be identified, $a$ and $b$ are the constant to be identified. The most leastsquares estimation of $\boldsymbol{\Phi}$ is $\hat{\boldsymbol{\Phi}}=\left(\mathbf{B}^{\mathrm{T}} \mathbf{B}\right)^{-1} \mathbf{B}^{\mathrm{T}} \mathbf{Y}$.

The discrete solution in the whitening differential Equation $\frac{\mathrm{d} x^{(1)}}{\mathrm{d} t}+a x^{(1)}=b$ is

$$
\hat{x}^{(1)}\left(t_{k}\right)=\frac{\hat{b}}{\hat{a}}+\left(x^{(1)}\left(t_{1}\right)-\frac{\hat{b}}{\hat{a}}\right) e^{-a\left(t_{k}-t_{1}\right)}(k=1,2, \cdots, m) .
$$

After restoring the reciprocal fitting value of the original data is

$$
\hat{x}^{(0)}\left(t_{k}\right)=\left\{\begin{array}{lc}
x^{(1)}\left(t_{1}\right), & (k=1), \\
\frac{x^{(1)}\left(t_{k}\right)-x^{(1)}\left(t_{k-1}\right)}{\Delta t_{k}}, & (k=2,3, \cdots, m),
\end{array}\right.
$$

Accounting to Definition 1, the model value of the original sequence is $\hat{x}^{(00)}\left(t_{k}\right)(k=1,2, \cdots, m)$.

The absolute error of the fitting data is

$$
q\left(t_{k}\right)=\hat{x}^{(00)}\left(t_{k}\right)-x^{(00)}\left(t_{k}\right) .
$$

The relative error of the fitting data (\%) is

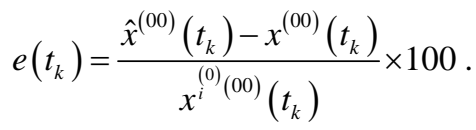

The mean of the relative error of the fitting data column:

$$
f=\frac{1}{m} \sum_{k=1}^{m}\left|e_{i}(k)\right| \text {. }
$$

After obtaining some data such as the simulation value, the predicted value and the error in non-equidistant $\operatorname{GRM}(1,1)$, the model can be tested [1]. The calculating program NGRM_I1.0 was compiled with Matlab [10].

\section{Example}

P. G. Foleiss researched that there is the influence of the temperature on fatigue strength under the long life symmetry cycle of many materials. Table 1 shows the experimental data of the change relation of Ti alloy fatigue strength along with temperature, which is a sequence of non-equidistant spacing. The data in $[2,3]$ were modeled by using the method proposed in this paper and we obtained the following result:

$$
\begin{aligned}
& a=-0.00097435, \quad b=0.001774, \\
& \hat{x}^{(1)}\left(t_{k}\right)=1.8225 e^{0.00097435(t-100)}-1.8207 .
\end{aligned}
$$

The fitting value of the original data is

$$
\begin{aligned}
\hat{\boldsymbol{\sigma}}_{-1}(\mathbf{T})= & {[560,554.9509,536.3301,515.8293,} \\
& \text { 498.5487, 484.1868, 467.9404, 452.2641, } \\
& \text { 437.0888]. }
\end{aligned}
$$

The absolute error of the fitting data is

$$
\begin{aligned}
\mathbf{q}(\mathbf{T})= & {[0,2.5891,-0.23009,0.27073,7.0513,} \\
& 1.9132,-0.54039,1.5359,-0.68884] .
\end{aligned}
$$

The relative error of the fitting data(\%)is

$$
\begin{aligned}
\mathbf{e}(\mathbf{T})= & {[0.46437,-0.042919,0.052457,1.3946,} \\
& 0.39358,-0.11562,0.33844,-0.1578] .
\end{aligned}
$$

The mean of the relative error of the fitting data column is $0.32887 \%$.

The mean relative error in the non-homogeneous model based on traditional accumulated generating in [4] is $0.33666 \%$.

After the original data were pre-processed by using $\mathbf{t}=\frac{\mathbf{T}-50}{50}$ and $\mathbf{X}^{(0)}=\frac{\boldsymbol{\sigma}_{-1}-400}{50}$ in [2], the maximum relative error is $4.86 \%$ and the mean relative error is $3.19 \%$. The model was established by using the function transformation method in [3] and the mean relative error is $0.6587 \%$. Homogeneous exponent function fitting onetime accumulated generating sequence was used in [5] and it is $0.9765 \%$. Thus, the examples validate the adaptability and the scientific of the proposed model.

\section{Conclusion}

In this paper, applying the reciprocal accumulated generating and the reconstruction method of GRM(1,1) model's

Table 1. Relation of Ti alloy fatigue strength $\left(\sigma_{-1}\right)$ along with temperature $(T)$.

\begin{tabular}{cccccc}
\hline No. & 1 & 2 & 3 & 4 & 5 \\
\hline $\mathrm{T}$ & 100 & 130 & 170 & 210 & 240 \\
$\sigma_{-1}$ & 560 & 557.54 & 536.10 & 516.10 & 505.60 \\
\hline \multicolumn{6}{c}{} \\
\hline No. & 6 & 7 & 8 & 9 \\
\hline $\mathrm{T}$ & 270 & 310 & 340 & 380 \\
$\sigma_{-1}$ & 486.1 & 467.4 & 453.8 & 436.4 \\
\hline
\end{tabular}


background value of non-equidistant sequence based on the exponential trait of grey model and the integral definition, a novel non-equidistant GRM(1,1) model based on reciprocal accumulated generating was put forward. The Matlab program of this model was written. This model can be used in non-equidistant interval \& equidistant interval time series and has the characteristic of high precision as well as high adaptability. Example validates the correctness and validity of the proposed model. There are important practical and theoretical significance and this model should be widely used.

\section{REFERENCES}

[1] Y. X. Luo, L. T. Zhang and M. Li, "Grey Systems and Applications in Mechanical Engineering,” National University of Defense Technology Press, Changsha, 2001.

[2] Y. X. Luo and J. R. Zhou, "Non-Equidistance GM(1,1) Model and Its Application in Fatigue Experimental Data Processing and On-Line Control," Journal of Mechanical Strength, Vol. 18, No. 3,1996, pp. 60-63.

[3] Y. X. Luo, X. Wu and M. Li, "Function-Transfer Method of Parameters Estimation Of Grey GM(1,1) Model and Its Application,” Journal of Mechanical Strength, Vol. 24, No. 3, 2002, pp. 450-452.
[4] W. Z. Dai and J. F. Li, "Modeling Research on Non-Equidistance GM(1,1) Model,” Systems Engineering Theory \& Practice, Vol. 25, No. 9, 2005, pp. 89-93 .

[5] F. X. Wang, "Improvement on Unequal Interval Gray Forecast Model,” Fuzzy Information and Engineering, Vol. 6, No. 1, 2006, pp.118-123.

[6] Y. M. Wang, Y. G. Dang and Z. X. Wang, "The Optimization of Background Value in Non-Equidistant GM(1,1) Model," Chinese Journal of Management Science, Vol. 16, No. 4, 2008, pp. 159-162.

[7] Z. M. Song and J. L. Deng, "The Accumulated Generating Operation in Opposite Direction and Its Use in Grey Model GOM(1,1)," Systems Engineering, Vol. 19, No. 1, 2001, pp. 66-69.

[8] B. H. Yang and Z. Q. Zhang, "The Grey Model Has Been Accumulated Generating Operation in Reciprocal Number and Its Application," Mathematics in Practice and Theory, Vol. 33, No. 10, 2003, pp. 21-25.

[9] H. Zhou and X. G. Wang, "A Improvement of the Grey Model GRM(1,1) Generated by Accumulation Generating Operation of Reciprocal Number," Journal of Shenyang Ligong University, Vol. 27, No. 4, 2008, pp. 84-86.

[10] D. Y. Xue and Y. Q. Chen, "Solving Applied Mathematical Problems with Matlab,” CRC Press, Inc., Outside North America, 2009. 